\title{
Uses of 4, 5, 6, 7-tetrahydrobenzo[b]thiophene Derivatives in Heterocyclic Synthesis: Synthesis of Pyrazol, Pyrimidine and Pyridazine Derivatives with Antimicrobial Activities
}

\author{
Wagnat W. Wardakhan* and Mastoura M. Edrees \\ National Organization for Drug Control and Research, P.O. \\ 29, Cairo, Egypt.
}

\begin{abstract}
7 HE 2-DAIZO-4,5,6,7-tetrahydrobenzo[b]thiophene-3-carboxamide coupled with either malononitrile or ethyl cyanoacetate to give the hydrazo derivatives $4 \mathrm{a}$ and $4 \mathrm{~b}$, respectively. The latter products underwent hetero-cyclization to give pyrazole, pyrimidine and pyridazine derivatives. The antimicrobial evaluation of the newly synthesized products was measured and most of the products showed interesting activities.
\end{abstract}

Keywords: Tetrahydrobenzo[b]thiophene, Pyrazole, Pyrimidine and Pyridazine.

Benzothiophenes are one of the most common and consequently the most studied classes of aromatic heterocycles ${ }^{(1)}$. The occurrence of these heterocycles in a significant number of medicinal agents ${ }^{(2)}$, active in a variety of disease areas, has led to an enduring interest in the development of new methods for their synthesis ${ }^{(3-5)}$. Methods that utilize new classes of precursors are particularly valuable. Among the many syntheses available, transition metal catalyzed processes $^{(6)}$ and palladiummediated methods in particular $^{(7)}$ feature heavily. Syntheses based on palladium-catalyzed cyclizations of appropriately substituted alkenyl or alkynyl phenols, or thiophenols, are versatile and have been used on many occasions ${ }^{(8-10)}$. Related tandem processes involving catalyzed $\mathrm{C}-\mathrm{C}$ bond formation, usually employing Sonogashira-type reactions, before construction of the key $\mathrm{C}-\mathrm{S}$ bond have also been developed ${ }^{(11-14)}$. The crucial bond-forming event in these processes is intramolecular of a nucleophilic oxygen or sulfur atom onto a palladium-activated $\mathrm{C}-\mathrm{C}$ multiple bond, resulting in formation of the $\mathrm{X}-\mathrm{C} 2$ bond of the heterocycle. We aim to develop an alternative method for the synthesis of 2-amino-3-anilido-4,5,6,7-tetrahydrobenzo[b]thiophene derivatives and their uses in heterocyclic synthesis together with their evaluation for antimicrobial and antifungal activities. 


\section{Results and Discussions}

\section{Chemistry}

The reaction between cyano acetanilide, cyclohexanone and elemental sulfur in ethanol solution containing triethylamine gave the 2-amino-3-anilido-4,5,6,7tetrahydrobenzo[b]thiophene $(1)^{(15)}$. The structure of compound 1 was based on the analytical and spectral data. The 2-amino group present in compound 1 underwent ready diazotization through the reaction of compound 1 with sodium nitrite in an acid medium at $0-5{ }^{\circ} \mathrm{C}$ to give the non-isolable intermediate 2-diazo3-(4-methy)-anilido-4,5,6,7-tetrahydrobenzo[b]thiophene (2). The reaction of the diazonium salt $2 a, b$ with either malononitrile (3a) or ethyl cyanoacetate (3b) in basic ethanol solution at $0{ }^{\circ} \mathrm{C}$ gave the hydrazone derivatives $4 \mathrm{a}$,b. The structures of $4 \mathrm{a}, \mathrm{b}$ were based on analytical and spectral data. Thus, the ${ }^{1} \mathrm{H}$ NMR spectrum of $4 \mathrm{a}$ showed the presence of two multiplets at d 1.36-1.60 and 2.622.68 for $4 \mathrm{CH}_{2}$, a singlet at $\mathrm{d} 2.88$ for $\mathrm{CH}_{3}$ group, a multiplet at $\mathrm{d}$ 7.21-7.35 for $\mathrm{C}_{6} \mathrm{H}_{4}$, two singlet $\left(\mathrm{D}_{2} \mathrm{O}\right.$ exchangeable) at $\mathrm{d} 8.80,9.30$ two $\mathrm{NH}$ groups.
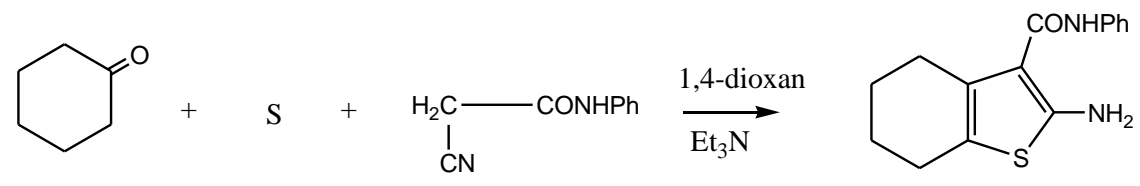<smiles></smiles><smiles></smiles>

2

Scheme 1

Compounds $4 \mathrm{a}, \mathrm{b}$ react with either hydrazine hydrate $5 \mathrm{a}$ or phenyl hydrazine $5 \mathrm{~b}$ to give the pyrazole derivatives 6a-d. Analytical and spectral data of the reaction products are consistent with the assigned structures (see experimental section). On the other hand, the reaction of $4 a, b$ with either urea (7a) or thiourea (7b) gave the pyrimidine derivatives $8 \mathrm{a}-\mathrm{d}$. The structures of the latter products were established on the basis of analytical and spectral data. Thus, ${ }^{1} \mathrm{H}$ NMR

Egypt. J. Chem. 53, No. 2 (2010) 
spectrum of 8a showed two multiplets at d 1.33-1.49 and 2.60-2.68 for $4 \mathrm{CH}_{2}$, a singlet at $\mathrm{d} 2.86$ for $\mathrm{CH}_{3}$ group, two singlet at $\mathrm{d} 4.80,5.41$ for two $\mathrm{NH}_{2}$ groups, a singlet $\left(\mathrm{D}_{2} \mathrm{O}\right.$ exchangeable) at $\mathrm{d} 8.77 \mathrm{NH}$ groups, a singlet at $\mathrm{d} 9.73$ for $\mathrm{OH}$ group. Compound $4 \mathrm{~b}$ capable for amide formations. Thus, its reaction with aniline in 1,4-dioxan solution gave the anilide derivative 9.

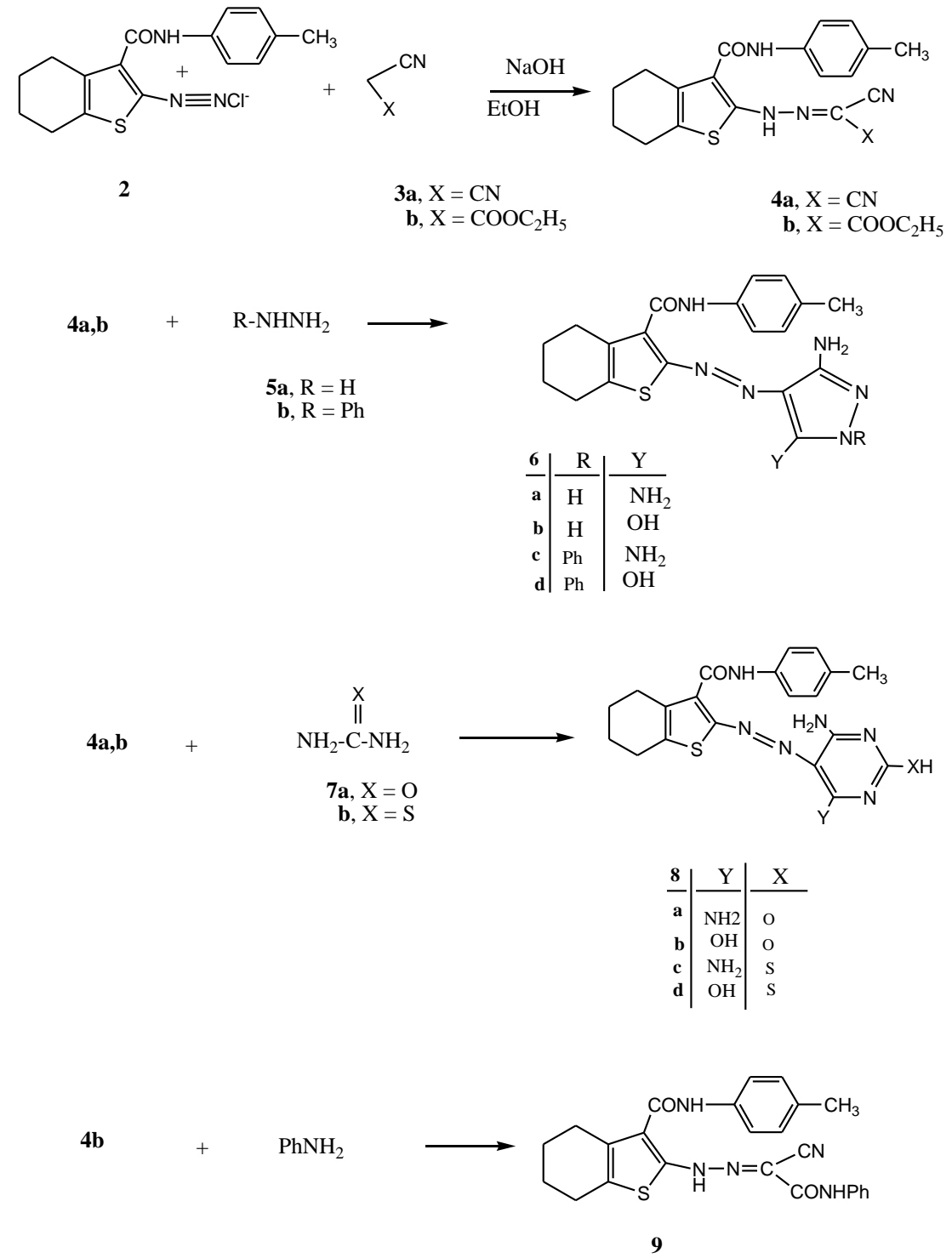

Scheme 2

Egypt. J. Chem. 53, No. 2 (2010) 
Compounds $4 \mathrm{a}$,b reacted with either malononitrile (3a) or ethyl cyanoacetate (3b) to give the pyridazine derivatives $11 \mathrm{a}-\mathrm{d}$. The analytical and spectral data of the reaction products are in analogy with the proposed structures (see experimental section). The reaction of either compound $4 \mathrm{a}$ or $4 \mathrm{~b}$ with phenylisothiocyanate (12) afforded the corresponding 1,2,4-triazine derivatives $13 \mathrm{a}$ and $13 \mathrm{~b}$, respectively.<smiles>[X]/C(C)=N/Nc1sc2c(c1C(N)=O)CCCC2</smiles>

4a, $\mathrm{X}=\mathrm{CN}$ b, $\mathrm{X}=\mathrm{COOC}_{2} \mathrm{H}_{5}$<smiles>[X]CC#N</smiles>
3a, $\mathrm{X}=\mathrm{CN}$ b, $\mathrm{X}=\mathrm{COOC}_{2} \mathrm{H}_{5}$<smiles>[X]CC(=N)N(N=C(C)C)c1sc2c(c1C(N)=O)CCC(C)C2</smiles>

10a-d<smiles>[X]c1c(C#N)nn(-c2sc3c(c2C(N)=O)CCCC3)c(=N)c1[X]</smiles>

\begin{tabular}{c|c|c}
$\mathbf{1 1}$ & $\mathrm{X}$ & $\mathrm{Y}$ \\
\hline $\mathbf{a}$ & $\mathrm{CN}$ & $\mathrm{NH}_{2}$ \\
$\mathbf{b}$ & $\mathrm{CN}$ & $\mathrm{OH}$ \\
c & $\mathrm{COOC}_{2} \mathrm{H}_{5}$ & $\mathrm{NH}_{2}$ \\
d & $\mathrm{COOC}_{2} \mathrm{H} 5$ & $\mathrm{OH}$
\end{tabular}

4a,b $+\quad$ PhNCS

12<smiles></smiles>

13a, $\mathrm{Y}=\mathrm{NH}$

b, $\mathrm{Y}=\mathrm{O}$

Scheme 3

Egypt. J. Chem. 53, No. 2 (2010) 
Test solutions were added to the inoculated wells, one control well on each slide being treated with solvent only. The slides were then returned to the incubator until germ tubes $400 \mathrm{~mm}( \pm 50 \mathrm{~mm})$ long were visible in the control wells. Further growth was arrested by the addition of lactophenol aniline blue to each of the wells. The minimal inhibitory concentration (MIC in $\mathrm{mg} \mathrm{ml}^{-1}$ ) was determined.

\section{Antimicrobial Activity}

The in vitro antimicrobial activity of the heterocyclic derivatives 1-13b against two strains of Gram positive bacteria (Bacillus subtilis CECT 498 and Bacillus cereus CECT 148), two strains of Gram negative bacteria (Escherichia coli ECT 101 and Pseudomonas aeruginosa) and Candida albicans CECT 1394 as a representative species of fungi was investigated. The newly synthesized products were dissolved in aqueous ethanol to give a logarithmic series of concentrations from 2 to $256 \mathrm{mg} / \mathrm{l}$ upon tenfold dilution with the growth medium and spore suspension of the test fungi. The toxicity of compounds was determined via a pipette additions into the wells of multi-well slides, followed with $25 \mu \mathrm{l}$ of the culture medium. The inoculated slides were then incubated at $25^{\circ} \mathrm{C}$ until short germ tubes appeared; approximately $50 \mathrm{~mm}$ in length (at $0 \mathrm{hr}$ ) was measured. Five $\mu \mathrm{l}$ volumes of the prepared compound adaptation of agar streak dilution method based on radial diffusion ${ }^{(16,17)}$. Under the same conditions ampicillin (antibacterial) and cycloheximide (antifungal) were used as standards. The MIC was considered to be the lowest concentration of the tested compound (in dimethylformamide) which inhibits growth of bacteria or fungi on the plate (Table 1). The diameters of the inhibition zones corresponding to the MICs are also presented in Table 1.

\section{Experimental}

\section{Chemistry}

All melting points are uncorrected. IR spectra were recorded for $(\mathrm{KBr})$ discs on a Pye Unicam SP-1000 spectrophotometer. ${ }^{1} \mathrm{H}$ NMR and ${ }^{13} \mathrm{C}$ NMR spectra were measured on a Varian EM 390-200 MHz in DMSOd6 as solvent and using TMS as internal standard, and chemical shifts are expressed as $\delta \mathrm{ppm}$. Analytical data were recorded at the Micro Analytical Data Unit at Cairo University, Giza, Egypt. 
TABLE 1. Antimicrobial activities of the tested compounds .

\begin{tabular}{|c|c|c|c|c|}
\hline \multicolumn{2}{|l|}{ Compound } & \multicolumn{3}{|c|}{ MIC gml $\left({ }^{-1}\right)$ (zone of inhibition, $\mathrm{mm}$ ) } \\
\hline \multicolumn{2}{|c|}{ E. coli $\mathrm{ECT}$} & B. cereus $\mathrm{CECT}$ & B. subtilis CECT & C. albicans CECT \\
\hline 1 & - & $6.05(15)$ & $21.01(8)$ & 33.23 \\
\hline $4 a$ & $12.50(6)$ & $22.52(8)$ & $20.55(4)$ & $8.65(4)$ \\
\hline $4 b$ & - & $22.38(8)$ & $3.72(6)$ & $16.58(12)$ \\
\hline $6 b$ & - & $11.32(3)$ & $18.22(8)$ & $12.40(4)$ \\
\hline $6 c$ & - & $19.15(4)$ & $20.46(9)$ & $0.40(10)$ \\
\hline $6 \mathrm{~d}$ & - & $18.12(5)$ & $12.25(2)$ & $23.64(6)$ \\
\hline $8 \mathrm{a}$ & $16.20(6)$ & $18.23(5)$ & $22.45(8)$ & 100.00 \\
\hline $8 b$ & - & $0.03(9)$ & $4.13(10)$ & $0.61(6)$ \\
\hline $8 \mathrm{c}$ & - & $10.05(6)$ & $16.42(2)$ & $4.55(10)$ \\
\hline $8 \mathrm{~d}$ & - & $18.24(7)$ & $6.18(4)$ & $0.40(5)$ \\
\hline 9 & - & $16.22(3)$ & $18.32(8)$ & $14.40(4)$ \\
\hline $11 \mathrm{a}$ & - & $10.38(4)$ & $6.22(6)$ & $12.55(12)$ \\
\hline $11 \mathrm{~b}$ & - & $6.25(15)$ & $22.01(8)$ & $30.23(6)$ \\
\hline $11 \mathrm{c}$ & - & $21.15(4)$ & $23.16(9)$ & 100.00 \\
\hline $11 \mathrm{~d}$ & - & $25.36(8)$ & $23.63(6)$ & $26.12(3)$ \\
\hline $13 \mathrm{a}$ & - & $0.08(9)$ & $2.13(10)$ & $0.85(6)$ \\
\hline $13 b$ & - & $12.05(6)$ & $14.42(2)$ & $4.66(10)$ \\
\hline Ampicillin & 6.25 & 3.13 & $12.50(10)$ & - \\
\hline Cycloheximide & $e-$ & - & - & $12.50(6)$ \\
\hline
\end{tabular}

2-Amino-4,5,6,7-tetrahydrobenzo[b] thiophene-3-N-phenylcarboxamide (1)

To a solution of cyanoacetanilide $(16.0 \mathrm{~g}, 0.01 \mathrm{~mol})$ in 1,4-dioxan $(80 \mathrm{ml})$ containing triethylamine $(1.0 \mathrm{ml})$ each of cyaclohexanone $(9.8 \mathrm{~g}, 0.01 \mathrm{~mol})$ and elemental sulphur $(0.32 \mathrm{~g}, 0.01 \mathrm{~mol})$ were added. The reaction mixture was heated under reflux for $2 \mathrm{~h}$ then poured onto ice/water containing few drops of hydrochloric acid.

1: Yellow crystals, yield 88\% (23.93), m.p. $146-148{ }^{\circ} \mathrm{C}$, (acetic acid). IR: $\left(\mathrm{v} / \mathrm{cm}^{-1}\right)=$ 3458-3241 (NH); $3042(\mathrm{CH}), 2899\left(\mathrm{CH}_{2}\right), 1710(\mathrm{C}=\mathrm{O}), 1638(\mathrm{C}=\mathrm{C}) .{ }^{1} \mathrm{HNMR}$ :

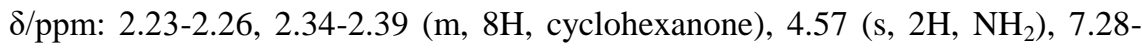
$7.36\left(\mathrm{~m}, 5 \mathrm{H}, \mathrm{C}_{6} \mathrm{H}_{5}\right), 8.93$ (s, $\left.1 \mathrm{H}, \mathrm{NH}\right) . \mathrm{C}_{15} \mathrm{H}_{16} \mathrm{~N}_{2} \mathrm{OS}$ (272.37), Calcd: C, 66.15; H, 5.92; N, 10.29; S, 11.77. Found: C, 66.01; H, 5.69; N, 10.51; A, 10.94.

Egypt. J. Chem. 53, No. 2 (2010) 
2- Hydrazodicyanomethino-3-(4- methylphenyl)- 4,5,6,7- tetrahydrobenzo [b]thiophene -3-(4-methylphenyl) carboxamide (4a) and 2-Hydrazoethylcyanoacetato3-(4-methylphenyl)- 4,5,6,7-tetrahydrobenzo[b]-thiophene-3-(4-methylphenyl) carboxamide (4b)

General procedure

To a cold solution $\left(0{ }^{\circ} \mathrm{C}\right)$ of $1(2.87 \mathrm{~g}, 0.01 \mathrm{~mol})$ in acetic acid $(20 \mathrm{ml})$, propanoic acid $(5 \mathrm{ml})$ and hydrochloric acid $(5 \mathrm{ml})$ sodium nitrite solution $(1.4 \mathrm{~g}$, $0.02 \mathrm{~mol}$ ) was added. The reaction mixture was stirred for 10 minutes then added to a solution of either malononitrile $(0.66 \mathrm{~mol}, 0.01 \mathrm{~mol})$ or ethyl cyanoacetate $(1.13 \mathrm{~g}, 0.01 \mathrm{~mol})$ in ethanol $(30 \mathrm{ml})$ containing sodium hydroxide $(5 \mathrm{~mL}, 20 \%)$ with continuous stirring. The formed solid product was collected by filtration and washed several times with water.

4a: Red crystals from ethanol, $70 \%$ yield $(2.55 \mathrm{~g}), \mathrm{mp} 154{ }^{\circ} \mathrm{C}, \mathrm{IR}\left(\mathrm{u}, \mathrm{cm}^{-1}\right)$ : 3460-3341 (2 NH), 2983, $2877\left(\mathrm{CH}_{3}, \mathrm{CH}_{2}\right), 2222,2220(2 \mathrm{CN}), 1684(\mathrm{C}=\mathrm{O})$, $1638(\mathrm{~N}=\mathrm{C}) .{ }^{1} \mathrm{H}$ NMR $(\mathrm{d} \mathrm{ppm}): 1.36-1.60\left(\mathrm{~m}, 4 \mathrm{H}, 2 \mathrm{CH}_{2}\right), 2.62-2.68(\mathrm{~m}, 4 \mathrm{H}$, $\left.2 \mathrm{CH}_{2}\right), 2.88\left(\mathrm{~s}, 3 \mathrm{H}, \mathrm{CH}_{3}\right), 7.21-7.35\left(\mathrm{~m}, 4 \mathrm{H}, \mathrm{C}_{6} \mathrm{H}_{4}\right), 8.80,9.30(2 \mathrm{~s}, 2 \mathrm{H}, 2 \mathrm{NH})$. $\mathrm{C}_{19} \mathrm{H}_{17} \mathrm{~N}_{5} \mathrm{OS}$ (363.44): $\mathrm{C}, 62.79 ; \mathrm{H}, 4.71 ; \mathrm{N}, 19.27 ; \mathrm{S}, 8.82$. Found: $\mathrm{C}, 62.69 ; \mathrm{H}$, $4.49 ; \mathrm{N}, 19.05 ; \mathrm{S}, 9.03$.

4b: Red crystals from ethanol, $77 \%$ yield $(3.15 \mathrm{~g}), \mathrm{mp} \quad 190{ }^{\circ} \mathrm{C}$, IR $\left(\mathrm{u}, \mathrm{cm}^{-1}\right)$ : 3478-3349 (2 NH), 2980, $2892\left(\mathrm{CH}_{3}, \mathrm{CH}_{2}\right), 2223(\mathrm{CN}), 1687,1682(2 \mathrm{C}=\mathrm{O})$, $1634(\mathrm{~N}=\mathrm{C}) .{ }^{1} \mathrm{H}$ NMR $\left(\mathrm{d}\right.$ ppm): $1.13\left(\mathrm{t}, 3 \mathrm{H}, \mathrm{J}=7.11 \mathrm{~Hz}, \mathrm{CH}_{3}\right), 1.33-1.62(\mathrm{~m}, 4 \mathrm{H}$, $\left.2 \mathrm{CH}_{2}\right), 2.60-2.67\left(\mathrm{~m}, 4 \mathrm{H}, 2 \mathrm{CH}_{2}\right), 2.85\left(\mathrm{~s}, 3 \mathrm{H}, \mathrm{CH}_{3}\right), 4.23(\mathrm{q}, 2 \mathrm{H}, \mathrm{J}=7.11 \mathrm{~Hz}$, $\mathrm{CH}_{2}$ ), 7.28-7.39 (m, $\left.4 \mathrm{H}, \mathrm{C}_{6} \mathrm{H}_{4}\right), 8.83,9.28(2 \mathrm{~s}, 2 \mathrm{H}, 2 \mathrm{NH}) . \mathrm{C}_{21} \mathrm{H}_{22} \mathrm{~N}_{4} \mathrm{O}_{3} \mathrm{~S}(410.49)$ : C, 61.44; H, 5.40; N, 13.65; S, 7.81. Found: C, 61.79; H, 5.59; N, 13.91; S, 8.03.

2- Azo(3, 5- diaminopyrazolo)-3-(4- methylphenyl) -4,5,6,7- tetrahydrobenzo-[b]thiophene-3-(4-methylphenyl)carboxamide (6a), 2-azo(3-amino-5-hydroxypyrazolo)3-(4-methylphenyl)-4,5,6,7- tetrahydrobenzo[b]-thiophene -3- (4-methylphenyl) carboxamide (6b), 2-azo(3,5-diamino-1-phenylpyrazolo)-3-(4-methylphenyl)-4,5,6,7tetrahydrobenzo[b]-thiophene-3-(4-methylphenyl)carboxamide (6c) and 2-azo(3amino5-hydroxy-1-phenylpyrazolo)-3-(4-methylphenyl)-4,5,6,7-tetrahydrobenzo [b]thiophene-3-(4-methylphenyl)carboxamide (6d)

General procedure

To a solution of either $3 \mathrm{a}(3.63 \mathrm{~g}, 0.01 \mathrm{~mol})$ or $4 \mathrm{~b}(4.10 \mathrm{~g}, 0.01 \mathrm{~mol})$ in ethanol $(50 \mathrm{ml})$ either hydrazine hydrate $(0.50 \mathrm{~g}, 0.01 \mathrm{~mol})$ or phenyl hydrazine $(1.08 \mathrm{~g}, 0.01 \mathrm{~mol})$ was added. The reaction mixture was heated under reflux for 2 $\mathrm{hr}$ then left to cool. The solid product formed upon pouring onto ice/water containing few drops of hydrochloric acid was collected by filtration. 
6a: Yellow crystals from ethanol, $67 \%$ yield $(2.67 \mathrm{~g}), \mathrm{mp} 205^{\circ} \mathrm{C}$, IR $\left(\mathrm{u}, \mathrm{cm}^{-1}\right)$ : 3483-3327 (2 NH, 2NH $), 2980,2889\left(\mathrm{CH}_{3}, \mathrm{CH}_{2}\right), 1687(\mathrm{C}=\mathrm{O}), 1635(\mathrm{C}=\mathrm{C}) .{ }^{1} \mathrm{H}$ NMR (d ppm): 1.33-1.58 (m, 4H, 2CH $\mathrm{CH}_{2}, 2.64-2.69\left(\mathrm{~m}, 4 \mathrm{H}, 2 \mathrm{CH}_{2}\right), 2.86(\mathrm{~s}, 3 \mathrm{H}$, $\left.\mathrm{CH}_{3}\right), 5.20\left(\mathrm{~s}, 4 \mathrm{H}, 2 \mathrm{NH}_{2}\right), 7.28-7.39\left(\mathrm{~m}, 4 \mathrm{H}, \mathrm{C}_{6} \mathrm{H}_{4}\right), 8.78,9.87(2 \mathrm{~s}, 2 \mathrm{H}, 2 \mathrm{NH})$. $\mathrm{C}_{19} \mathrm{H}_{21} \mathrm{~N}_{7} \mathrm{OS}$ (395.48): C, 57.70; H, 5.35; N, 24.79; S, 8.11. Found: C, 57.93; H, $5.76 ; \mathrm{N}, 25.06 ; \mathrm{S}, 7.93$.

6b: Pale yellow crystals from ethanol, $55 \%$ yield $(2.17 \mathrm{~g}), \mathrm{mp} 204{ }^{\circ} \mathrm{C}$, IR (u, $\left.\mathrm{cm}^{-1}\right)$ : 3520-3322 (OH, $\left.2 \mathrm{NH}, \mathrm{NH}_{2}\right), 2983,2886\left(\mathrm{CH}_{3}, \mathrm{CH}_{2}\right), 1689(\mathrm{C}=\mathrm{O}), 1635$ $(\mathrm{C}=\mathrm{C}) .{ }^{1} \mathrm{H}$ NMR $(\mathrm{d} \mathrm{ppm}): 1.34-1.57\left(\mathrm{~m}, 4 \mathrm{H}, 2 \mathrm{CH}_{2}\right), 2.63-2.68\left(\mathrm{~m}, 4 \mathrm{H}, 2 \mathrm{CH}_{2}\right)$, $2.87\left(\mathrm{~s}, 3 \mathrm{H}, \mathrm{CH}_{3}\right), 5.21\left(\mathrm{~s}, 2 \mathrm{H}, \mathrm{NH}_{2}\right) 7.30-7.38\left(\mathrm{~m}, 4 \mathrm{H}, \mathrm{C}_{6} \mathrm{H}_{4}\right), 8.66,9.91(2 \mathrm{~s}, 2 \mathrm{H}$, $2 \mathrm{NH}), 10.25$ (s, br, 1H, OH). $\mathrm{C}_{19} \mathrm{H}_{20} \mathrm{~N}_{6} \mathrm{O}_{2} \mathrm{~S}$ (396.47): C, 57.56; H, 5.08; N, 21.20; S, 8.09. Found: C, 57.38; H, 5.25; N, 21.05; S, 7.88.

6c: Yellow crystals from 1,4-dioxan, $71 \%$ yield $(3.33 \mathrm{~g}), \mathrm{mp} \quad 168-170{ }^{\circ} \mathrm{C}, \mathrm{IR}$ $\left(\mathrm{u}, \mathrm{cm}^{-1}\right)$ : 3486-3352 (NH, 2NH$), 2986,2890\left(\mathrm{CH}_{3}, \mathrm{CH}_{2}\right), 1686(\mathrm{C}=\mathrm{O}), 1633$ $(\mathrm{C}=\mathrm{C}) .{ }^{1} \mathrm{H}$ NMR $(\mathrm{d} \mathrm{ppm}): 1.35-1.59\left(\mathrm{~m}, 4 \mathrm{H}, 2 \mathrm{CH}_{2}\right), 2.58-2.69\left(\mathrm{~m}, 4 \mathrm{H}, 2 \mathrm{CH}_{2}\right)$, $2.89\left(\mathrm{~s}, 3 \mathrm{H}, \mathrm{CH}_{3}\right), 5.34\left(\mathrm{~s}, 4 \mathrm{H}, 2 \mathrm{NH}_{2}\right), 7.32-7.36\left(\mathrm{~m}, 9 \mathrm{H}, \mathrm{C}_{6} \mathrm{H}_{4}\right), 8.69$ (s, H, NH). $\mathrm{C}_{25} \mathrm{H}_{25} \mathrm{~N}_{7} \mathrm{OS}$ (471.58): C, 63.67; H, 5.34; N, 20.79; S, 6.80. Found: C, 63.58; H, $5.60 ; \mathrm{N}, 20.94 ; \mathrm{S}, 7.25$.

6d: Orange crystals from 1,4-dioxan, $63 \%$ yield $(2.99 \mathrm{~g}), \mathrm{mp} 235^{\circ} \mathrm{C}, \mathrm{IR}\left(\mathrm{u}, \mathrm{cm}^{-1}\right)$ : 3520-3324 (OH, NH, $\left.\mathrm{NH}_{2}\right), 2983,2884\left(\mathrm{CH}_{3}, \mathrm{CH}_{2}\right), 1686(\mathrm{C}=\mathrm{O}), 1636(\mathrm{C}=\mathrm{C})$. ${ }^{1} \mathrm{H}$ NMR (d ppm): 1.30-1.55 (m, 4H, 2 $\left.\mathrm{CH}_{2}\right), 2.60-2.69\left(\mathrm{~m}, 4 \mathrm{H}, 2 \mathrm{CH}_{2}\right), 2.86$ (s, $\left.3 \mathrm{H}, \mathrm{CH}_{3}\right), 5.34\left(\mathrm{~s}, 2 \mathrm{H}, \mathrm{NH}_{2}\right), 7.28-7.39\left(\mathrm{~m}, 9 \mathrm{H}, \mathrm{C}_{6} \mathrm{H}_{4}\right), 8.66$ (s, H, NH), 10.22 (s, $1 \mathrm{H}, \mathrm{OH}) . \mathrm{C}_{25} \mathrm{H}_{24} \mathrm{~N}_{6} \mathrm{O}_{2} \mathrm{~S}$ (472.56): C, 63.54; H, 5.12; N, 17.78; S, 6.79. Found: $\mathrm{C}$, 63.64; H, 5.05; N, 17.90; S, 7.03.

2-Azo(4,6-diamino-2-hydroxypyrimidino)-3-(4-methylphenyl)-4,5,6,7-tetrahydrobenzo[b]-thiophene-3-(4-methylphenyl) carboxamide (8a), 2-azo(4-amino -2,6dihydroxypyrimidino)-3-(4-methylphenyl)-4,5,6,7-tetrahydrobenzo[b]-thiophene-3-(4methylphenyl) carboxamide (8b), 2-azo(4,6-diamino-2-mercaptopyrimidino)-3-(4methylphenyl)-4,5,6,7-tetrahydrobenzo[b]-thiophene-3-(4-methylphenyl) carboxamide (8c) and 2-azo(4-amino-6-hydroxy-2-mercaptopyrimidino)-3-(4-methylphenyl)-4,5,6,7tetrahydrobenzo[b]-thiophene-3-(4-methylphenyl)carboxamide (8d)

General procedure

To a suspension of either $4 \mathrm{a}(3.63 \mathrm{~g}, 0.01 \mathrm{~mol})$ or $4 \mathrm{~b}(4.10 \mathrm{~g}, 0.01 \mathrm{~mol})$ in sodium ethoxide solution [prepared by dissolving sodium metal $(0.46 \mathrm{~g}, \quad 0.02$ $\mathrm{mol})$ in absolue ethanol $(30 \mathrm{ml})]$ either urea $(0.60 \mathrm{~g}, 0.01 \mathrm{~mol})$ or thiourea $(0.76$ $\mathrm{g}, 0.01 \mathrm{~mol}$ ) was added. The reaction mixture was heated in a boiling water bath 
for $3 \mathrm{hr}$ then poured onto ice/water then poured onto ice/water and neutralized with a few drops of hydrochloric acid (till $\mathrm{pH} 6$ ).

8a: Orange crystals from acetic acid, $70 \%$ yield $(2.96 \mathrm{~g}), \mathrm{mp} 172{ }^{\circ} \mathrm{C}$, IR (u, $\left.\mathrm{cm}^{-1}\right)$ : 3490-3342 (OH, NH, 2NH $)_{2}, 2977,2890\left(\mathrm{CH}_{3}, \mathrm{CH}_{2}\right), 1689(\mathrm{C}=\mathrm{O}), 1638$ $(\mathrm{C}=\mathrm{C}) .{ }^{1} \mathrm{H}$ NMR (d ppm): $1.35-1.59\left(\mathrm{~m}, 4 \mathrm{H}, 2 \mathrm{CH}_{2}\right), 2.62-2.68\left(\mathrm{~m}, 4 \mathrm{H}, 2 \mathrm{CH}_{2}\right)$, $2.90\left(\mathrm{~s}, 3 \mathrm{H}, \mathrm{CH}_{3}\right), 4.72,5.07\left(2 \mathrm{~s}, 4 \mathrm{H}, 2 \mathrm{NH}_{2}\right), 7.32-7.36\left(\mathrm{~m}, 4 \mathrm{H}, \mathrm{C}_{6} \mathrm{H}_{4}\right), 8.80(\mathrm{~s}$, $1 \mathrm{H}, \mathrm{NH}), 9.02$ (s, $1 \mathrm{H}, \mathrm{OH}) . \mathrm{C}_{20} \mathrm{H}_{21} \mathrm{~N}_{7} \mathrm{O}_{2} \mathrm{~S}$ (423.49): C, 56.72; H, 5.00; N, 23.15; S, 7.57. Found: C, 56.79; H, 4.83; N, 23.07; S, 7.80.

8b: Yellow crystals from ethanol, $66 \%$ yield $(2.79 \mathrm{~g}), \mathrm{mp} \quad 215^{\circ} \mathrm{C}$, IR $\left(\mathrm{u}, \mathrm{cm}^{-1}\right)$ : 3538-3356 (2OH, NH, $\left.\mathrm{NH}_{2}\right), 2978,2889\left(\mathrm{CH}_{3}, \mathrm{CH}_{2}\right), 1683(\mathrm{C}=\mathrm{O}), 1638(\mathrm{C}=\mathrm{C})$. ${ }^{1} \mathrm{H}$ NMR (d ppm): 1.36-1.60 (m, 4H, 2CH $\left.\mathrm{CH}_{2}\right), 2.62-2.66\left(\mathrm{~m}, 4 \mathrm{H}, 2 \mathrm{CH}_{2}\right), 2.90(\mathrm{~s}$, $\left.3 \mathrm{H}, \mathrm{CH}_{3}\right), 5.08\left(\mathrm{~s}, 2 \mathrm{H}, \mathrm{NH}_{2}\right) 7.29-7.36\left(\mathrm{~m}, 4 \mathrm{H}, \mathrm{C}_{6} \mathrm{H}_{4}\right), 8.68(\mathrm{~s}, 1 \mathrm{H}, \mathrm{NH}), 9.68$, $10.22(2 \mathrm{~s}, 2 \mathrm{H}, 2 \mathrm{OH}) . \mathrm{C}_{20} \mathrm{H}_{20} \mathrm{~N}_{6} \mathrm{O}_{3} \mathrm{~S}$ (424.48): C, 56.59; H, 4.75; N, 19.80; S, 7.55. Found: C, 56.84; H, 4.99; N, 20.31; S, 7.70.

8c: Pale brown crystals from acetic acid, $62 \%$ yield $(2.72 \mathrm{~g}), \mathrm{mp} 142-148^{\circ} \mathrm{C}, \mathrm{IR}$ $\left(\mathrm{u}, \mathrm{cm}^{-1}\right)$ : 3456-3320 (NH, 2NH $), 2982,2893\left(\mathrm{CH}_{3}, \mathrm{CH}_{2}\right), 1688(\mathrm{C}=\mathrm{O}), 1630$ $(\mathrm{C}=\mathrm{C}) .{ }^{1} \mathrm{H}$ NMR $(\mathrm{d} \mathrm{ppm}): 1.35-1.60\left(\mathrm{~m}, 4 \mathrm{H}, 2 \mathrm{CH}_{2}\right), 2.58-2.66\left(\mathrm{~m}, 4 \mathrm{H}, 2 \mathrm{CH}_{2}\right)$, $2.86\left(\mathrm{~s}, 3 \mathrm{H}, \mathrm{CH}_{3}\right), 3.01$ (s, br, $\left.1 \mathrm{H}, \mathrm{SH}\right), 4.86,5.24\left(2 \mathrm{~s}, 4 \mathrm{H}, 2 \mathrm{NH}_{2}\right), 7.30-7.38(\mathrm{~m}$, $\left.4 \mathrm{H}, \mathrm{C}_{6} \mathrm{H}_{4}\right), 8.70$ (s, H, NH). $\mathrm{C}_{20} \mathrm{H}_{21} \mathrm{~N}_{7} \mathrm{OS}_{2}$ (439.56): C, 54.65; H, 4.82; N, 22.31; S, 14.59. Found: C, 54.82; H, 4.91; N, 22.47; S, 14.89 .

8d: Yellow crystals from ethanol, $66 \%$ yield $(2.90 \mathrm{~g}), \mathrm{mp} 173{ }^{\circ} \mathrm{C}$, IR $\left(\mathrm{u}, \mathrm{cm}^{-1}\right)$ : 3346-3336 (OH, $\left.2 \mathrm{NH}, \mathrm{NH}_{2}\right), 2977,2892\left(\mathrm{CH}_{3}, \mathrm{CH}_{2}\right), 1688(\mathrm{C}=\mathrm{O}), 1634(\mathrm{C}=\mathrm{C})$. ${ }^{1} \mathrm{H}$ NMR (d ppm): 1.32-1.58 (m, 4H, 2 $\left.\mathrm{CH}_{2}\right), 2.58-2.67$ (m, 4H, 2 $\left.\mathrm{CH}_{2}\right), 2.87$ (s, $\left.3 \mathrm{H}, \mathrm{CH}_{3}\right), 3.31$ (s, $\left.1 \mathrm{H}, \mathrm{SH}\right), 5.23\left(\mathrm{~s}, 2 \mathrm{H}, \mathrm{NH}_{2}\right), 7.32-7.38\left(\mathrm{~m}, 4 \mathrm{H}, \mathrm{C}_{6} \mathrm{H}_{4}\right), 8.70(\mathrm{~s}$, $\mathrm{H}, \mathrm{NH}), 10.03$ (s, $1 \mathrm{H}, \mathrm{OH}) . \mathrm{C}_{20} \mathrm{H}_{20} \mathrm{~N}_{6} \mathrm{O}_{2} \mathrm{~S}_{2}$ (440.54): C, 54.53; H, 4.58; N, 19.08; S, 14.56. Found: C, 54.82; H, 4.80; N, 18.83; S, 14.74.

2- hydrazo(cyanoacetanilide) -3- (4-methylphenyl) -4,5,6,7-tetrahydrobenzo-[b]thiophene-3-(4-methylphenyl)carboxamide (9)

To a solution $4 \mathrm{~b}(4.10 \mathrm{~g}, 0.01 \mathrm{~mol})$ in 1,4-dioxan $(30 \mathrm{ml})$, aniline $(0.94 \mathrm{~g}$, $0.01 \mathrm{~mol}$ ) was added. The reaction mixture was heated under reflux for $1 \mathrm{hr}$ then evaporated under vacuum. The remaining product was triturated with ethanol and the formed solid product was collected by filtration.

9: Orange crystals from acetic acid, $50 \%$ yield $(2.28 \mathrm{~g}), \mathrm{mp} 207^{\circ} \mathrm{C}$, IR $\left(\mathrm{u}, \mathrm{cm}^{-1}\right)$ : 3432-3338 (3NH), 2978, $2890\left(\mathrm{CH}_{3}, \mathrm{CH}_{2}\right), 2222 \mathrm{CN}, 1691,1688$ (2 C=O), 1633 $(\mathrm{C}=\mathrm{C}) .{ }^{1} \mathrm{H}$ NMR $(\mathrm{d} \mathrm{ppm}): 1.33-1.60\left(\mathrm{~m}, 4 \mathrm{H}, 2 \mathrm{CH}_{2}\right), 2.56-2.67\left(\mathrm{~m}, 4 \mathrm{H}, 2 \mathrm{CH}_{2}\right)$, $2.87\left(\mathrm{~s}, 3 \mathrm{H}, \mathrm{CH}_{3}\right), 7.28-7.38\left(\mathrm{~m}, 9 \mathrm{H}, \mathrm{C}_{6} \mathrm{H}_{4}, \mathrm{C}_{6} \mathrm{H}_{5}\right), 8.68,8.88-9.02(3 \mathrm{~s}, 3 \mathrm{H}, 3 \mathrm{NH})$. 
$\mathrm{C}_{25} \mathrm{H}_{23} \mathrm{~N}_{5} \mathrm{O}_{2} \mathrm{~S}$ (457.55): C, 65.63; H, 5.07; N, 15.31; S, 7.01. Found: C, 65.93; H, $4.91 ; \mathrm{N}, 15.03 ; \mathrm{S}, 6.88$.

2-(4-Amino-3,5-dicyano-6-iminopyridazino)-3-(4-methylphenyl)-4,5,6,7-tetrahydrobenzo -[b]-thiophene-3-(4-methylphenyl)carboxamide (11a), 2-(3,5-dicyano-3-hydroxy-6iminopyridazino) -3-(4-methylphenyl) -4,5,6,7-tetrahydrobenzo [b]- thiophene-3-(4methylphenyl) carboxamide (11b), 2- (4-amino -3- cyano -5- ethoxycarbonyl-6iminopyridazino )-3- (4-methylphenyl) -4, 5, 6, 7-tetrahydrobenzo [b]- thiophene -3(4-methylphenyl)carboxamide (11c) and 2-(3-cyano-4-hydroxy-5-hydroxycarbonyl6-iminopyridazino)-3-(4-methylphenyl)-4,5,6,7-tetrahydrobenzo[b]-thiophene-3-(4methylphenyl)carboxamide (11d)

General procedure

To a solution of either $4 \mathrm{a}(3.63 \mathrm{~g}, 0.01 \mathrm{~mol})$ or $4 \mathrm{~b}(4.10 \mathrm{~g}, 0.01 \mathrm{~mol})$ in $1,4-$ dioxan $(40 \mathrm{ml})$ containing triethylamine $(0.5 \mathrm{ml})$ either malononitrile $(0.66 \mathrm{~g}$, $0.01 \mathrm{~mol})$ or ethyl cyanoacetate $(1.13 \mathrm{~g}, 0.01 \mathrm{~mol})$ was added. The reaction mixture was heated under reflux for $2 \mathrm{~h}$ then left to cool. The solid product formed upon pouring onto ice/water was collected by filtration.

11a: Buff crystals from acetic acid, $80 \%$ yield $(3.43 \mathrm{~g}), \mathrm{mp} \quad 207^{\circ} \mathrm{C}$, IR $\left(\mathrm{u}, \mathrm{cm}^{-1}\right)$ : 3466-3352 (2 NH, NH$), 2980,2877\left(\mathrm{CH}_{3}, \mathrm{CH}_{2}\right), 2220,2222(2 \mathrm{CN}), 1687(\mathrm{C}=\mathrm{O})$, $1666(\mathrm{C}=\mathrm{N}), 1634(\mathrm{C}=\mathrm{C}) .{ }^{1} \mathrm{H}$ NMR $(\mathrm{d} \mathrm{ppm}): 1.32-1.59\left(\mathrm{~m}, 4 \mathrm{H}, 2 \mathrm{CH}_{2}\right), 2.60-2.67$ (m, $\left.4 \mathrm{H}, 2 \mathrm{CH}_{2}\right), 2.87\left(\mathrm{~s}, 3 \mathrm{H}, \mathrm{CH}_{3}\right), 4.70\left(\mathrm{~s}, 2 \mathrm{H}, \mathrm{NH}_{2}\right), 7.28-7.38\left(\mathrm{~m}, 4 \mathrm{H}, \mathrm{C}_{6} \mathrm{H}_{4}\right)$, 8.23, $8.76(2 \mathrm{~s}, 2 \mathrm{H}, 2 \mathrm{NH}) . \mathrm{C}_{22} \mathrm{H}_{19} \mathrm{~N}_{7} \mathrm{OS}$ (429.50): C, 61.52; H, 4.46; N, 22.83; S, 7.47. Found: C, $61.49 ; \mathrm{H}, 4.51 ; \mathrm{N}, 23.02 ; \mathrm{S}, 7.65$.

11b: Orange crystals from ethanol, $89 \%$ yield $(3.81 \mathrm{~g}), \mathrm{mp} 148{ }^{\circ} \mathrm{C}, \mathrm{IR}\left(\mathrm{u}, \mathrm{cm}^{-1}\right)$ : 3530-3321(OH, 2NH), 2983, $2891\left(\mathrm{CH}_{3}, \mathrm{CH}_{2}\right), 2225,00(2 \mathrm{CN}), 1677(\mathrm{C}=\mathrm{O})$, $1635(\mathrm{C}=\mathrm{C}) .{ }^{1} \mathrm{H}$ NMR $(\mathrm{d} \mathrm{ppm}): 1.33-1.58\left(\mathrm{~m}, 4 \mathrm{H}, 2 \mathrm{CH}_{2}\right), 2.63-2.69(\mathrm{~m}, 4 \mathrm{H}$, $2 \mathrm{CH}_{2}$ ), $2.92\left(\mathrm{~s}, 3 \mathrm{H}, \mathrm{CH}_{3}\right), 7.32-7.38\left(\mathrm{~m}, 4 \mathrm{H}, \mathrm{C}_{6} \mathrm{H}_{4}\right), 8.58,9.04(2 \mathrm{~s}, 2 \mathrm{H}, 2 \mathrm{NH})$, 10.20 (s, $1 \mathrm{H}, \mathrm{OH}) . \mathrm{C}_{22} \mathrm{H}_{18} \mathrm{~N}_{6} \mathrm{O}_{2} \mathrm{~S}$ (430.48): C, 61.38; H, 4.21; N, 19.52; $\mathrm{S}, 7.45$. Found: C, 61.47; H, 4.37; N, 19.84; S, 7.52.

11c: Brown crystals from acetic acid, $77 \%$ yield $(3.66 \mathrm{~g}), \mathrm{mp} \quad 187{ }^{\circ} \mathrm{C}$, IR (u, $\left.\mathrm{cm}^{-1}\right)$ : 3444-3329 (2NH, NH$), 2986,2890\left(\mathrm{CH}_{3}, \mathrm{CH}_{2}\right), 2222(\mathrm{CN}), 1692,1687$ $(2 \mathrm{C}=\mathrm{O}), 1629(\mathrm{C}=\mathrm{C}) .{ }^{1} \mathrm{H}$ NMR (d ppm): $1.13\left(\mathrm{t}, 3 \mathrm{H}, \mathrm{J}=7.02 \mathrm{~Hz}, \mathrm{CH}_{3}\right), 1.33-$ $1.58\left(\mathrm{~m}, 4 \mathrm{H}, 2 \mathrm{CH}_{2}\right), 2.49-2.63\left(\mathrm{~m}, 4 \mathrm{H}, 2 \mathrm{CH}_{2}\right), 2.89\left(\mathrm{~s}, 3 \mathrm{H}, \mathrm{CH}_{3}\right), 4.22(\mathrm{q}, 2 \mathrm{H}, \mathrm{J}=$ $\left.7.02 \mathrm{~Hz}, \mathrm{CH}_{2}\right), 4.89\left(\mathrm{~s}, 2 \mathrm{H}, \mathrm{NH}_{2}\right), 7.29-7.36\left(\mathrm{~m}, 4 \mathrm{H}, \mathrm{C}_{6} \mathrm{H}_{4}\right), 8.68,9.06(2 \mathrm{~s}, 2 \mathrm{H}$, 2NH). $\mathrm{C}_{24} \mathrm{H}_{24} \mathrm{~N}_{6} \mathrm{O}_{3} \mathrm{~S}$ (476.55): C, 60.49; H, 5.08; N, 17.64; S, 6.73. Found: C, $60.53 ; \mathrm{H}, 4.88 ; \mathrm{N}, 17.47 ; \mathrm{S}, 6.99$. 
11d: Orange crystals from 1,4-dioxan, $50 \%$ yield $(2.38 \mathrm{~g}), \mathrm{mp} \quad 195{ }^{\circ} \mathrm{C}$, IR $(\mathrm{u}$, $\left.\mathrm{cm}^{-1}\right)$ : 3365-3324 (OH, $\left.2 \mathrm{NH}\right), 2982,2888\left(\mathrm{CH}_{3}, \mathrm{CH}_{2}\right), 2220(\mathrm{CN}), 1689,1680$ $(2 \mathrm{C}=\mathrm{O}), 1636(\mathrm{C}=\mathrm{C}) .{ }^{1} \mathrm{H}$ NMR $(\mathrm{d} p p m): 1.12\left(\mathrm{t}, 3 \mathrm{H}, \mathrm{J}=6.73 \mathrm{~Hz}, \mathrm{CH}_{3}\right), 1.32-$ $1.61\left(\mathrm{~m}, 4 \mathrm{H}, 2 \mathrm{CH}_{2}\right), 2.55-2.66\left(\mathrm{~m}, 4 \mathrm{H}, 2 \mathrm{CH}_{2}\right), 2.89\left(\mathrm{~s}, 3 \mathrm{H}, \mathrm{CH}_{3}\right), 4.22(\mathrm{q}, 2 \mathrm{H}, \mathrm{J}=$ $\left.7.02 \mathrm{~Hz}, \mathrm{CH}_{2}\right), 7.30-7.39\left(\mathrm{~m}, 4 \mathrm{H}, \mathrm{C}_{6} \mathrm{H}_{4}\right), 8.79,9.05(2 \mathrm{~s}, 2 \mathrm{H}, 2 \mathrm{NH}), 10.06(\mathrm{~s}, 1 \mathrm{H}$, OH). $\mathrm{C}_{24} \mathrm{H}_{23} \mathrm{~N}_{5} \mathrm{O}_{4} \mathrm{~S}$ (477.54): C, 60.36; H, 4.85; N, 14.67; S, 6.71. Found: $\mathrm{C}$, $60.51 ; \mathrm{H}, 4.89 ; \mathrm{N}, 14.85 ; \mathrm{S}, 6.90$.

2-(6-cyano-5-imino-3-thiono- 1-pheny-1,2,3- triazin- 2-yl)-4,5,6,7- tetrahydro-benzo[b]-thiophene-3-(4-methylphenyl) carboxamide (13a) and 2-(6-cyano-5-imino-3thiono-1-pheny-1,2,3-triazin-2-yl)-4,5,6,7-tetrahydro-benzo-[b]- thiophene -3- (4methylphenyl) carboxamide (13b)

General procedure

To a solution of either $4 \mathrm{a}(3.63 \mathrm{~g}, 0.01 \mathrm{~mol})$ or $4 \mathrm{~b}(4.10 \mathrm{~g}, 0.01 \mathrm{~mol})$ in $1,4-$ dioxan $(40 \mathrm{ml})$ containing triethylamine $(0.5 \mathrm{ml})$ phenylisothiocyanate $(1.30 \mathrm{~g}$, $0.01 \mathrm{~mol}$ ) was added. The whole mixture was heated under reflux for $4 \mathrm{hr}$ then poured onto ice/water containing few drops of hydrochloric acid and the formed solid product was collected by filtration.

13a: Yellow crystals from ethanol, $50 \%$ yield $(2.49 \mathrm{~g}), \mathrm{mp} 160^{\circ} \mathrm{C}$, IR $\left(\mathrm{u}, \mathrm{cm}^{-1}\right)$ : 3443-3329 (2NH), 2980, $2864\left(\mathrm{CH}_{3}, \mathrm{CH}_{2}\right), 2227(\mathrm{CN}), 1688(\mathrm{C}=\mathrm{O}), 1641(\mathrm{C}=\mathrm{C})$, 1205-1190 (C=S). ${ }^{1} \mathrm{H}$ NMR (d ppm): 1.38-1.60 (m, 4H, 2 $\left.2 \mathrm{CH}_{2}\right), 2.48-2.66(\mathrm{~m}, 4 \mathrm{H}$, $\left.2 \mathrm{CH}_{2}\right), 2.83$ (s, $\left.3 \mathrm{H}, \mathrm{CH}_{3}\right), 7.27-7.33\left(\mathrm{~m}, 9 \mathrm{H}, \mathrm{C}_{6} \mathrm{H}_{5}, \mathrm{C}_{6} \mathrm{H}_{4}\right), 8.83,9.32(2 \mathrm{~s}, 2 \mathrm{H}$, $2 \mathrm{NH}$ ). $\mathrm{C}_{26} \mathrm{H}_{22} \mathrm{~N}_{6} \mathrm{OS}_{2}$ (498.62): C, 62.63; H, 4.45; N, 16.85; S, 12.86. Found: $\mathrm{C}$, $62.41 ; \mathrm{H}, 4.61 ; \mathrm{N}, 17.15 ; \mathrm{S}, 13.09$.

13b: Yellow crystals from ethanol, $50 \%$ yield $(2.49 \mathrm{~g}), \mathrm{mp} \quad 135 \quad{ }^{\circ} \mathrm{C}$, IR (u, $\left.\mathrm{cm}^{-1}\right)$ : 3448-3326 (NH), 2980, $2893\left(\mathrm{CH}_{3}, \mathrm{CH}_{2}\right), 2227(\mathrm{CN}), 1689,1683(2 \mathrm{C}=\mathrm{O})$, $1636(\mathrm{C}=\mathrm{C}), 1203-1194(\mathrm{C}=\mathrm{S}) .{ }^{1} \mathrm{H}$ NMR $(\mathrm{d} \mathrm{ppm}): 1.28-1.55\left(\mathrm{~m}, 4 \mathrm{H}, 2 \mathrm{CH}_{2}\right)$, 2.60-2.68 (m, $\left.4 \mathrm{H}, 2 \mathrm{CH}_{2}\right), 2.88\left(\mathrm{~s}, 3 \mathrm{H}, \mathrm{CH}_{3}\right), 7.24-7.36\left(\mathrm{~m}, 9 \mathrm{H}, \mathrm{C}_{6} \mathrm{H}_{5}, \mathrm{C}_{6} \mathrm{H}_{4}\right), 8.90$ (s, $1 \mathrm{H}, \mathrm{NH}$ ). $\mathrm{C}_{26} \mathrm{H}_{21} \mathrm{~N}_{5} \mathrm{O}_{2} \mathrm{~S}_{2}$ (499.61): C, 62.50; H, 4.24; N, 14.02; S, 12.84. Found: C, 62.74; H, 4.49; N, 13.93; S, 13.21.

\section{References}

1. Pozharskii, A.F. Soldatenkov, A.T. and Katritzky, A.R., Heterocycles in Life and Society, Wiley, Chichester, UK (1997).

2. Queiroz, M. J. R. P., Ferreira, I. C. F. R., Calhelha, R. C. and Estevinho, L. M., Synthesis and antioxidant activity evaluation of new 7-aryl or 7-heteroarylamino-2,3dimethylbenzo $[b]$ thiophenes obtained by Buchwald-Hartwig $\mathrm{C}-\mathrm{N}$ cross-coupling. Bioorg. Med. Chem. 15 (4), 1788 (2007). 
3. Gilchrist, T. L., synthesis of aromatic heterocycles. J. Chem. Soc., Perkin Trans. 1, 2491 (2001).

4. Horton, D.A., Bourne, G.T. and Smythe, M.L., The combinatorial synthesis of bicyclic privileged structures or privileged substructures. Chem. Rev. 103, 893 (2003).

5. Donnelly, D. M. X. and Meegan, M. J. The structure, reactions, synthesis, and uses of heterocyclic compounds In: Comprehensive Heterocyclic Chemistry, A. R. Katritzky and C. W. Rees, Eds. Elsevier Science \& Technology, USA, Vol. 4, p. 657 (1984).

6. Nakamura, I. and Yamamoto, Y., Transition-metal-catalyzed reactions in heterocyclic synthesis. Chem. Rev. 104, 2127 (2004).

7. Li, J.J. and Gribble, G.W., Palladium in heterocyclic chemistry - A guide for the synthetic chemist. Palladium in Heterocyclic Chemistry, Pergamon, Amsterdam (2000).

8. Cacchi, S., Fabrizi, G. and Goggiomani, Palladium-catalyzed functionalization of C$\mathrm{H}$ bonds and alkenes. Heterocycles, 56, 613 (2002).

9. Cacchi, S., Palladium reagents and catalysts: new perspectives for the $21^{\text {st }}$ century. $J$. Organomet. Chem. 42, 576 (1999).

10. Cacchi, S. and Arcadi, A., In: Handbook of Organopalladium Chemistry for Organic Synthesis, E. I. Negishi, Ed. Wiley, New York, Vol. 2, p. 2193 (2002).

11. Pal, M., Subramanian, V. and Yeleswarapu, K.R., Palladium mediated stereospecific synthesis of 3-enynyl substituted. Tetrahedron Lett. 44, 8221 (2003).

12. Dai, W. M. and Lai, K.W. First synthesis of nitrobenzo[b]furans via a coupling cyclization approach: Wei-Min Dai and Kwong Wah Lai. Tetrahedron Lett. 43, 9377 (2002).

13. Flynn, B.L., Hamel, E. and Jung, M.K., One-pot synthesis of benzo[b]furan and indole inhibitors of tubulin polymerization. J. Med. Chem. 45, 2670 (2002).

14. Hu, Y. and Yang, Z., The palladium-mediated intramolecular carbonylative annulation of o-alkynylphenols to synthesisze beozo[b]furo[3,4-d]furan-1-ones. Org. Lett. 3, 1387 (2001).

15. Palitis, E., Gudriniece, E., Barkane, P., Rizh, and Politekh, R. Utilisation de dervatives del l'Isoondoline commeinsecticides. ademijas Vestis, Kimijas Serija,5, 633 (1986).

Egypt. J. Chem. 53, No. 2 (2010) 
16. Hawkey, P.M. and Lewis, A.A., Medical Bacteriology: A Practical Approach, Oxford University, Oxford, UK Press, pp. 181-194 (1994).

17. Rameshkumar, N.,Ashokkumar, M., Subramanian, E.H., Ilavarasan, E.R. and Sridhar, S.k., Synthesis of 6-fluoro-1,4-dihydro-4-oxo-quinoline-3-carboxylic acid derivatives as potential antimicrobial agents, Synthesis of 6-fluoro-1,4-dihydro-4-oxoquinoline-3-carboxylic acid derivatives as potential antimicrobial agents. Eur. J. Med. Chem. 38, 1001 (2003).

(Received 25/2/2010;

accepted 22/3/2010)

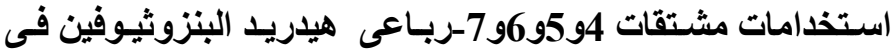

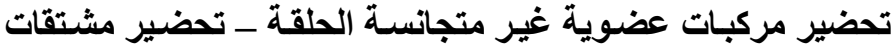

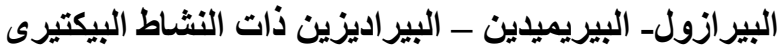

$$
\begin{aligned}
& \text { وجنات وهبة وردخان و مستورة محمد أدريس }
\end{aligned}
$$

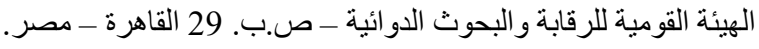

تتـاول هذا البحث تحضير مشتقات الثيوفين عن طريق استخدام مشتقات الديازو

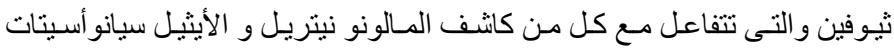

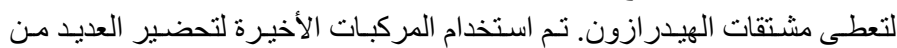

الكو اشف الكيميائية ليعطى مشتقات مختلفة من الثيوفين.

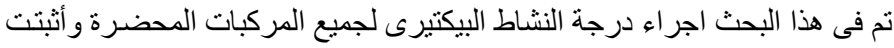

$$
\text { الدر اسة درجة نشاط عالية. }
$$

